\title{
Analytical investigation of properties of the iso-NTCP envelope
}

\author{
Pavel Stavrev ${ }^{2}$, Colleen Schinkel ${ }^{1,2}$, Nadia Stavreva ${ }^{2}$, Krassimir Markov $^{2}$, \\ B. Gino Fallone ${ }^{1-3}$
}

${ }^{1}$ Department of Physics, University of Alberta, Edmonton, Alberta, Canada; ${ }^{2}$ Department of Medical Physics, Cross Cancer Institute, 11560 University Ave, Edmonton, Alberta, T6G 1Z2, Canada; ${ }^{3}$ Department of Oncology, University of Alberta, Edmonton, Alberta, Canada

\begin{abstract}
Background. A property of the integral dose-volume histogram (DVH) space is analytically investigated in this work. A curve called an $\alpha$-iso-NTCP (normal tissue complication probability) envelope is constructed by connecting points belonging to step-like integral DVHs, each corresponding to homogeneous partial organ irradiation of a relative volume $v_{k}$ to dose $D_{k}$ such that the resulting NTCP has, in all cases, a particular value $\alpha$. The two subspaces into which the envelope divides the DVH space are analytically explored in terms of the equivalent uniform doses (EUDs) corresponding to the different DVHs. It is theoretically proven that any $\mathrm{DVH}$, other than the step-like $\mathrm{DVH}$, passing through a point $\left(D_{k}, v_{k}\right)$ from the $\alpha$-iso-NTCP envelope, will result in an NTCP $>\alpha$.

Conclusions. Thus, it is proven that any DVH that at least partially lies above the envelope results in an $N T C P>\alpha$. For some of the DVHs lying under the envelope, e.g. those that are tangential to the envelope, it is also true that the resulting NTCP $>\alpha$. However, it was numerically demonstrated elsewhere that there exist DVHs lying entirely in the lower subspace that result in an NTCP $<\alpha$. Therefore, one can conclude that since there is a chance that a DVH lying under the $\alpha$-iso-NTCP envelope will result in NTCP $<\alpha$, it would be preferable in the treatment optimization process to seek solutions for DVHs lying entirely under an iso-NTCP envelope and avoid solutions that have DVHs above an iso-NTCP envelope.
\end{abstract}

Key words: dose - response relationship, radiation; NTCP, iso-effect, DVH

\section{Introduction}

Recently we proposed that the DVH averaged from those resulting in a certain

Received 28 February 2007

Accepted 7 March 2007

Correspondence to: B. Gino Fallone, Ph.D., Department of Medical Physics, Cross Cancer Institute, 11560 University Ave., Edmonton, Alberta, T6G 1Z2, Canada; Phone: +1 780 432-8750; Fax: +1 780 432-8615; E-mail: gfallone@phys.ualberta.ca normal tissue complication probability (NTCP) can be used as a source of dosevolume constraints for inverse planning. ${ }^{1,2}$ Constraint points were estimated for a number of organs using two NTCP models - the Lyman model ${ }^{3}$ with the parameters of Burman et al. ${ }^{4}$ and the critical volume population model ${ }^{5}$ with the parameters of Stavrev et al. ${ }^{6}$ We also reported an observed property of the integral dose-volume histogram (DVH) space. ${ }^{1,2}$ In those reports we constructed a curve, which we called an 
$\alpha$-iso-NTCP envelope, by connecting points belonging to step-like integral DVHs. Each of these DVHs corresponded to homogeneous partial organ irradiation of a relative volume $v_{k}$ to dose $D_{k}$ such that, for each $\mathrm{DVH}$, the resulting NTCP had a particular value $\alpha$.

We numerically demonstrated that any DVH passing through a point $\left(D_{k}, v_{k}\right)$ from the $\alpha$-iso-NTCP envelope, i.e., any DVH that tangents or crosses the envelope, will result in an $N T C P \geq \alpha$. It should be emphasized that the equality is valid only for the step-like DVH that corresponds to the homogeneous partial organ irradiation of $v_{k}$ to $D_{k}$. In our present report, we prove, ana- lytically, this property of the $\alpha$-iso-NTCP envelope for the three most commonly used NTCP models - the Lyman model, the individual critical volume model and the population critical volume model.

\section{Proof for the Lyman model}

For our purposes, a normalized integral DVH is defined as a monotonically decreasing function characterized by the set of points $D_{i}, v_{i}: i=1, \ldots, N$ such that $v_{1}=1$, $v_{N+1}=0, v_{i+1}<v_{i}, D_{i}<D_{i+1}$.

We begin this proof for the Lyman $^{3}$ NTCP model:

$$
N T C P=\Phi\left(\frac{E U D-D_{50}}{m D_{50}}\right) ; \Phi(x)=\frac{1}{\sqrt{2 \pi}} \int_{-\infty}^{x} \exp \left(\frac{-t^{2}}{2}\right) d t=\frac{1}{2}\left[1+\operatorname{erf}\left(\frac{x}{\sqrt{2}}\right)\right]
$$

where $m$ and $D_{50}$ are model parameters, and EUD is the equivalent uniform dose, which will be defined later. It is clear from Eq. [1] that NTCP is a monotonically increasing function of EUD. Thus, for two arbitrary EUDs, if $E U D_{1}>E U D_{2}$, then it follows that NTCP $\left(E U D_{1}\right)>$ $\operatorname{NTCP}\left(\operatorname{EUD}_{2}\right)$.

Consider an arbitrary integral DVH, with points $\left(D_{i}, v_{i}: i=1, \ldots, N\right)$, that passes through the point $\left(D_{k}, v_{k}\right)$ on the $\alpha$-iso-NTCP envelope (see Figure 1). The EUD of this arbitrary DVH will be referred to as EUD. Now consider a step-like DVH that also passes through the same point. This step-like DVH has an NTCP of $\alpha$. If we call the EUD of this DVH EUD $\alpha_{\alpha}$, then we may write that $\operatorname{NTCP}\left(E U D_{\alpha}\right)=\alpha$. According to our observation, ${ }^{2}$ the NTCP of the arbitrary DVH that passes through a point on the $\alpha$-iso-NTCP envelope will be greater than $\alpha$ :

[2a] $N T C P(E U D)>N T C P\left(E U D_{\alpha}\right)=\alpha$.

Because of the monotonic nature of NTCP as a function of EUD, this statement is true, if and only if,

[2b] $E U D>E U D_{\alpha}$.

Therefore, if we can show that $E U D>E U D_{\alpha}$, then Eq. [2a] is also true.

To calculate EUD, the integral DVHs must be converted into differential DVHs. In the case of homogeneous partial organ irradiation of volume $v_{k}$ to a dose $D_{k}$, the integral and the differential DVHs are determined solely by the pair $\left(v_{k}, D_{k}\right)$. For any other type of irradiation, the corresponding differential DVH is given by the following set of points: $\left(v_{i}-v_{i+1}, D_{i}\right)$.

One of the commonly accepted forms of EUD is the one given by the generalized mean dose $(G M D)::^{7-9}$ 
[3] $E U D=G M D=\left(\sum_{i}\left(v_{i}-v_{i+1}\right) D_{i}^{1 / n}\right)^{n}$,

where $n$ is a volume parameter. For the case of partial organ irradiation of the volume $v_{k}$ to dose $D_{k}$, Eq. [3] simplifies to:

[4] $E U D_{\alpha}=\left(v_{k} D_{k}^{1 / n}\right)^{n}=v_{k}^{n} D_{k}$.

For the arbitrary DVH passing through point $\left(D_{k}, v_{k}\right)$, the EUD may be written as:

[5]

$$
E U D=\left(\sum_{i=1}^{N}\left(v_{i}-v_{i+1}\right) D_{i}^{1 / n}\right)^{n}
$$

$$
=\left(\sum_{i=1}^{k-1}\left(v_{i}-v_{i+1}\right) D_{i}^{1 / n}+\left(v_{k}-v_{k+1}\right) D_{k}^{1 / n}+\sum_{i=k+1}^{N}\left(v_{i}-v_{i+1}\right) D_{i}^{1 / n}\right)^{n}
$$

To prove Eq. [2b], we have to prove, from Eqs. [4] and [5], that the following inequality is valid:

$$
E U D_{\alpha}<E U D
$$

[6]

$$
\Rightarrow\left(v_{k} D_{k}^{1 / n}\right)^{n}<\left(\sum_{i=1}^{k-1}\left(v_{i}-v_{i+1}\right) D_{i}^{1 / n}+\left(v_{k}-v_{k+1}\right) D_{k}^{1 / n}+\sum_{i=k+1}^{N}\left(v_{i}-v_{i+1}\right) D_{i}^{1 / n}\right)^{n} .
$$

Taking each side of Eq. [6] to the power of $1 / \boldsymbol{n}$, we obtain:

[7] $v_{k} D_{k}^{1 / n}<\sum_{i=1}^{k-1}\left(v_{i}-v_{i+1}\right) D_{i}^{1 / n}+\left(v_{k}-v_{k+1}\right) D_{k}^{1 / n}+\sum_{i=k+1}^{N}\left(v_{i}-v_{i+1}\right) D_{i}^{1 / n}$,

which can then be written as:

$$
v_{k+1} D_{k}^{1 / n}<\sum_{i=1}^{k-1}\left(v_{i}-v_{i+1}\right) D_{i}^{1 / n}+\sum_{i=k+1}^{N}\left(v_{i}-v_{i+1}\right) D_{i}^{1 / n} .
$$

We now proceed by proving that Eq. [8] is true.

First, consider the term $v_{k+1} D_{k}^{1 / n}$ in Eq. [8]. It may be re-written as:

[9]

$$
v_{k+1} D_{k}^{1 / n}=\left(v_{k+1}-v_{k+2}\right) D_{k}^{1 / n}+\left(v_{k+2}-v_{k+3}\right) D_{k}^{1 / n}+\ldots+\left(v_{N}-v_{N+1}\right) D_{k}^{1 / n}
$$

$$
=\sum_{i=k+1}^{N}\left(v_{i}-v_{i+1}\right) D_{k}^{1 / n}
$$

where, by definition, $v_{N+1}=0$.

We can expand the second sum in Eq. [8]:

$$
\sum_{i=k+1}^{N}\left(v_{i}-v_{i+1}\right) D_{i}^{1 / n}=\left(v_{k+1}-v_{k+2}\right) D_{k+1}^{1 / n}+\left(v_{k+2}-v_{k+3}\right) D_{k+2}^{1 / n}+\ldots+\left(v_{N}-v_{N+1}\right) D_{N}^{1 / n}
$$

According to our definition of the integral DVH, $D_{i}<D_{i+1}$ for all $i=1 \ldots N$. Therefore, each term of the sum in Eq. [9] is less than the corresponding term in Eq. [10], and we can write: 


$$
v_{k+1} D_{k}^{1 / n}<\sum_{i=k+1}^{N}\left(v_{i}-v_{i+1}\right) D_{i}^{1 / n}
$$

Because of our definition of an integral DVH, $v_{i}>v_{i+1}$ for all $i=1 \ldots N$, the first sum in Eq. [8] is greater than zero:

[12] $\sum_{i=1}^{k-1}\left(v_{i}-v_{i+1}\right) D_{i}^{1 / n}>0$.

From Eqs. [11] and [12], the following is true:

$$
v_{k+1} D_{k}^{1 / n}<\sum_{i=1}^{k-1}\left(v_{i}-v_{i+1}\right) D_{i}^{1 / n}+\sum_{i=k+1}^{N}\left(v_{i}-v_{i+1}\right) D_{i}^{1 / n}
$$

which is identical to Eq. [8]. Thus, we have proven Eq. [8], which is equivalent to Eq. [6], and thus, Eq. [2b]. Therefore, Eq. [2a] is also true, and we have thus mathematically proven the property of the envelope for the Lyman model.

\section{Proof for the critical volume model}

The basic property of the $\alpha$-iso-NTCP envelope will also be proven for the critical volume (CV) NTCP model. The CV model exists in two forms - individual and population models. The individual CV model is given by:

$$
N T C P_{i n d}=\Phi\left[\frac{\sqrt{N}\left(\bar{\mu}_{d}-\mu_{c r}\right)}{\sigma_{\mu_{d}}}\right]
$$

where $N$ is the total number of functional subunits (FSUs) comprising the organ, $\bar{\mu}_{d}$ is the mean relative damaged volume, $\sigma_{\mu_{d}}$ is the variance in $\bar{\mu}_{d}$, and $\mu_{c r}$ is the relative critical volume of the organ. $5,10,11$

The population CV model, under the assumption that only the relative critical volume displays inter-patient variability, is given by:

$$
N T C P_{p o p}=\Phi\left[\frac{-\ln \left(-\ln \bar{\mu}_{d}\right)+\ln \left(-\ln \bar{\mu}_{c r}\right)}{-\sigma_{\mu_{c r}} / \bar{\mu}_{c r} \ln \bar{\mu}_{c r}}\right]
$$

where $\bar{\mu}_{c r}$ is the population mean relative critical volume and $\sigma_{\mu_{c r}}$ is the variance in $\bar{\mu}_{c r}{ }^{5}$

As can be seen in Eqs. [14] and [15], both the individual and the population CV models are monotonically increasing functions of the mean relative damaged volume $\bar{\mu}_{d}$. This quantity is given by the following sum:

[16] $\bar{\mu}_{d}=\sum_{i}\left(v_{i}-v_{i+1}\right) P_{F S U}\left(D_{i}\right)$,

where $P_{F S U}\left(D_{i}\right)$ is the probability that a functional subunit is damaged beyond repair. It, in turn, is given by:

$$
P_{F S U}\left(D_{i}\right)=\exp \left[-N_{c} \exp \left(-\alpha_{c} D_{i}\right)\right]
$$


where $N_{c}$ is the number of cells in an FSU and $\alpha_{c}$ is the cell radiosensitivity. The quantity $\exp \left(-\alpha_{c} D\right)$ is the probability that a cell survives an irradiation to dose $D$. Since $\alpha_{c}$ is a positive quantity, then $\exp \left(-\alpha_{c} D\right)$ is a decreasing function of dose. The term $N_{c} \exp \left(-\alpha_{c} D\right)$ is the mean number of cells that survive dose $D$ and also decreases as $D$ increases. Equation [17] is the probability that a functional subunit is damaged beyond repair, which is equivalent to the probability that all cells in the subunit are destroyed. Therefore, $\exp \left(-N_{c} e^{-\alpha_{c} D}\right)$, which is the probability of zero cell survivals, increases with decreasing mean number of cell survivals, $N_{c} \exp \left(-\alpha_{c} D\right)$, or increasing dose $D$.

We now compare the mean relative damaged volume caused by an arbitrary DVH that is tangential to or is crossing the $\alpha$-iso-NTCP envelope at point $\left(D_{k}, v_{k}\right)$ with the mean relative damaged volume caused by a step-like DVH given by $\left(D_{k}, v_{k}\right)$. From Eq. [16], the mean relative damaged volume for the arbitrary DVH passing through the point $\left(D_{k}, v_{k}\right)$ on the $\alpha$-iso-NTCP envelope is:

$$
\begin{aligned}
\bar{\mu}_{d} & =\sum_{i=1}^{N}\left(v_{i}-v_{i+1}\right) P_{F S U}\left(D_{i}\right) \\
& =\sum_{i=1}^{k-1}\left(v_{i}-v_{i+1}\right) P_{F S U}\left(D_{i}\right)+\left(v_{k}-v_{k+1}\right) P_{F S U}\left(D_{k}\right)+\sum_{i=k+1}^{N}\left(v_{i}-v_{i+1}\right) P_{F S U}\left(D_{i}\right)
\end{aligned} .
$$

The mean relative damaged volume caused by partial organ homogeneous irradiation of relative volume $v_{k}$ to dose $D_{k}$ will be denoted as $\bar{\mu}_{d, \alpha}$ and is given by:

[19] $\bar{\mu}_{d, \alpha}=v_{k} P_{F S U}\left(D_{k}\right)$.

We now compare $\bar{\mu}_{d}=\sum_{i=1}^{N}\left(v_{i}-v_{i+1}\right) P_{F S U}\left(D_{i}\right)$ (Eq. [18]), containing point $\left(D_{k}, v_{k}\right)$, with $\bar{\mu}_{d, \alpha}=v_{k} P_{F S U}\left(D_{k}\right)$ (Eq. [19]). Since $P_{F S U}(D)$ is an increasing function of dose, Eqs. [18] and [19] are similar to the EUD form of Eq. [3] from the Lyman model. By applying the same process as to the proof of Eq. [6] it can be shown that the following inequality is valid:

[20] $\bar{\mu}_{d}=\sum_{i=1}^{N}\left(v_{i}-v_{i+1}\right) P_{F S U}\left(D_{i}\right)>\bar{\mu}_{d, \alpha}=v_{k} P_{F S U}\left(D_{k}\right)$.

Given that NTCP is an increasing function of the mean relative damaged volume, it follows that $\operatorname{NTCP}\left(\bar{\mu}_{d}\right)>\operatorname{NTCP}\left(\bar{\mu}_{d, \alpha}\right)=\alpha$ for DVHs having a common point with the $\alpha$-isoNTCP envelope. 


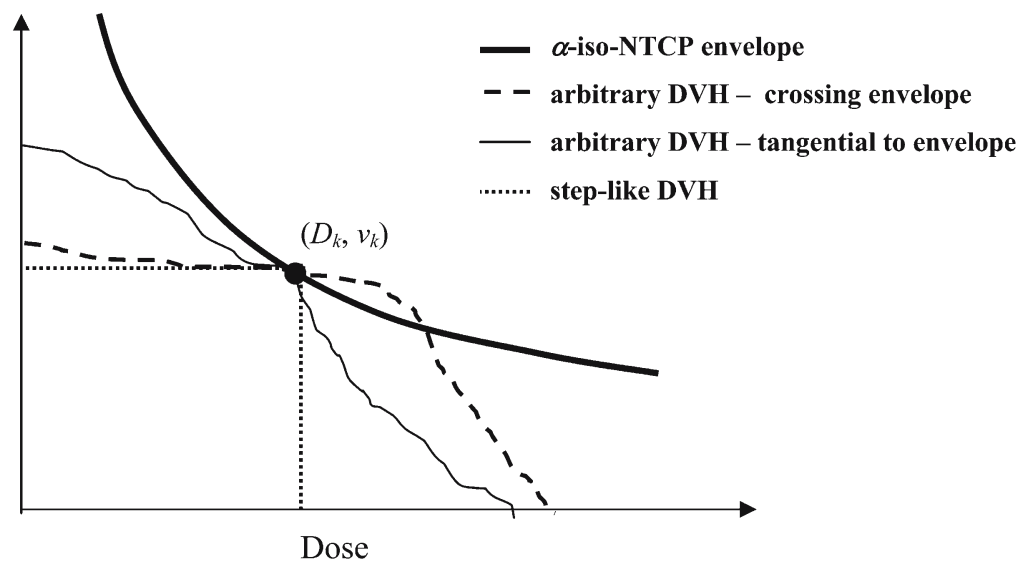

Figure 1. Illustration of an $\alpha$-iso-NTCP envelope and two arbitrary DVH curves - one that crosses the envelope at the point $\left(D_{k}, v_{k}\right)$ and one that is tangential to the envelope at the same point. Also shown is a step-like DVH passing through $\left(D_{k}, v_{k}\right)$ that corresponds to homogeneous partial organ irradiation. The NTCP of the step-like DVH should be equal to $\alpha$, while the NTCP for both arbitrary DVHs should be greater than $\alpha$.

\section{Discussion and conclusions}

Because we have proven that the discussed property of the $\alpha$-iso-NTCP envelope applies to three of the most commonly used NTCP models - the Lyman model, the critical volume individual model, and the critical volume population model - there is reason to believe that this property may be model-independent.

The $\alpha$-iso-NTCP envelope divides the dose-volume space in two sub-spaces. For the sub-space above the envelope, we have analytically proven that all DVH curves with at least one point in this region result in an NTCP $>\alpha$. For the sub-space under the $\alpha$-iso-NTCP envelope, it was numerically demonstrated elsewhere ${ }^{2}$ that there exist DVH curves that result in an NTCP < $\alpha$. However, as it is shown above, there do exist other curves, e.g., those that are tangential to the envelope from below, which result in an NTCP $>\alpha$. Nevertheless, since there is a chance that a DVH lying under the $\alpha$-iso-NTCP envelope will result in an
NTCP less than $\alpha$, one can conclude that it would be preferable in the treatment optimization process to seek solutions for DVHs lying entirely under an iso-NTCP envelope and avoid those that lie even partially above the envelope.

The physical dose-volume constraint points that we calculated in a previous work ${ }^{2}$ were found to be dependent on the NTCP model as well as the parameters used for their determination. The iso-NTCP envelope could be used to estimate the impact of a change of NTCP model and/or parameters on the calculated constraint points for a given organ, since the envelope curve is dependent on both of these quantities. Dawson et al. ${ }^{12}$ observed that the iso-NTCP curve corresponding to their liver parameters for the Lyman ${ }^{3}$ model was shifted considerably to the right in DVH space compared to the iso-NTCP curve corresponding to the Burman et al. ${ }^{4}$ parameters for the same organ. To estimate how the source of dose-volume constraints (the average of DVHs with a certain NTCP) would change 
with a change of NTCP parameter values, one could calculate the iso-NTCP envelope corresponding to these new parameters. The distance in DVH space between the old and new iso-NTCP curves is approximately the same as the distance between the old and new averaged DVHs. The position of the new dose-volume constraints could then be estimated by shifting them in DVH space by an amount equal to the distance between the two iso-NTCP curves. In this way, one can avoid having to perform an extensive recalculation of the dose-volume constraints.

\section{Acknowledgements}

This research was supported by studentships from the Alberta Foundation for Medical Research, the Alberta Cancer Board and the Translational Research Training in Cancer program (Canadian Institutes for Health Research), as well as the Alberta Cancer Board Research Initiative Program Grant RI-218/20624.

\section{References}

1. Schinkel-Ranger C, Stavrev P, Stavreva N, Weldon M, Scrimger R, Fallone BG. On the dose-volume constraints based on radiobiological considerations. Presented at the AAPM 47th Annual Meeting, Seattle, WA; 2005.

2. Schinkel C, Stavrev P, Stavreva N, Fallone BG. A theoretical approach to the problem of dose-volume constraint estimation and their impact on the dose-volume histogram selection. Med Phys 2006; 33: 3444-59.

3. Lyman JT. Complication probability as assessed from dose-volume histograms. Radiat Res Suppl 1985; 8: S13-19.

4. Burman C, Kutcher GJ, Emami B, Goitein M. Fitting of normal tissue tolerance data to an analytic function. Int J Radiat Oncol Biol Phys 1991; 21: 123-35.
5. Stavrev P, Stavreva N, Niemierko A, Goitein M. Generalization of a model of tissue response to radiation based on the idea of functional subunits and binomial statistics. Phys Med Biol 2001; 46: 1501-18.

6. Stavrev P, Stavreva N, Niemierko A, Goitein M. The application of biological models to clinical data. Phys Medica 2001; XVII: 71-82.

7. Niemierko A. Reporting and analyzing dose distributions: a concept of equivalent uniform dose. Med Phys 1997; 24: 103-10.

8. Niemierko A. A generalized concept of equivalent uniform dose. Presented at the 41th AAPM Annual Meeting, Nashville; 1999.

9. Stavrev P, Hristov D, Sham E. IMRT inverse treatment planning optimization based on physical constraints and biological objectives. Presented at the 47nd Annual General Meeting of the Canadian Organization of Medical Physicists (COMP), Kelowna, BC, Canada; 2001.

10. Jackson A, Kutcher GJ, Yorke ED. Probability of radiation-induced complications for normal tissues with parallel architecture subject to nonuniform irradiation. Med Phys 1993; 20: 613-25.

11. Niemierko A, Goitein M. Modeling of normal tissue response to radiation: the critical volume model. Int J Radiat Oncol Biol Phys 1993; 25: 135-45.

12. Dawson LA, Normolle D, Balter JM, McGinn CJ, Lawrence TS, Ten Haken RK, Analysis of radiation-induced liver disease using the Lyman NTCP model. Int J Radiat Oncol Biol Phys 2002; 53: 810-21. 